\title{
Análisis de la participación del café en las exportaciones agropecuarias en el Ecuador periodo 2015-2019
}

\section{Analysis of the participation of coffee in agricultural exports in Ecuador period 2015-2019}

DOI: $10.46932 / \mathrm{sfjdv2n4-057}$

Received in: May 1st, 2021

Accepted in: Jun 30th, 2021

\author{
Damaris Adelaida Banchón Cacao \\ Estudiante carrera de economía agropecuaria \\ Universidad Técnica de Machala. Km 5 1/2 vía Pasaje \\ Machala-Ecuador \\ E-mail: dbanchon_est@utmachala.edu.ec \\ Jessica Maribel Quezada Campoverde \\ Ingeniera agrónoma \\ Universidad Técnica de Machala. Km 5 1/2 vía Pasaje \\ Machala-Ecuador \\ E-mail: jquezada@utmachala.edu.ec \\ Eveligh Prado Carpio \\ Ingeniera comercial \\ Universidad Técnica de Machala. Km 5 1/2 vía Pasaje \\ Machala-Ecuador \\ E-mail: eprado@utmachala.edu.ec \\ Salomón Barrezueta Unda \\ Dr. en investigación agraria y forestal. PHD \\ Universidad Técnica de Machala. Km 5 1/2 vía Pasaje \\ Machala-Ecuador \\ E-mail: sabarrezueta@utmachala.edu.ec
}

\section{RESUMEN}

En el Ecuador la producción de café cumple un papel importante en la economía del país, siendo destacada a lo largo de los años, ya que es una de las fuentes importantes que genera empleo y divisas, por lo tanto, la presente investigación se desarrolló en Ecuador, que se encuentra ubicado en América del Sur, y tiene como objetivo "analizar la participación del café en las exportaciones agropecuarias del Ecuador en el periodo 2015-219”, mediante un enfoque descriptivo, de corte transversal y no experimental cuyo diseño es de tipo exploratorio, donde se evaluaron las cifras históricas del Sistema de Información y Producción Agropecuaria. Los resultados revelaron que la producción de café en Ecuador ha mantenido una tendencia decreciente en el periodo evaluado por diferentes causas como; las malas condiciones de las plantaciones por la afectación de la roya, precio del producto, tecnificación, factores climáticos, costos, escasez en mano de obra, vías de comunicación, y déficit en demanda del producto.

Palabras Clave: Exportaciones, participación, crecimiento. 


\begin{abstract}
In Ecuador, coffee production plays an important role in the country's economy, standing out over the years, since it is one of the important sources that generates employment and foreign exchange, therefore, this research was developed in Ecuador, which It is located in South America. , and aims to "analyze the participation of coffee in agricultural exports from Ecuador in the period 2015-219", through a descriptive, cross-sectional and non-experimental approach whose design is exploratory, where the historical figures of the Information Agricultural and production system was evaluated. The results revealed that coffee production in Ecuador has maintained a decreasing trend in the period evaluated for different causes such as; the poor conditions of the plantations due to the impact of rust, product price, modernization, climatic factors, costs, labor shortage, communication routes and deficit in product demand.
\end{abstract}

Keywords: Exports, participation, growth.

\title{
1 INTRODUCCIÓN
}

Ecuador es un país netamente agrícola que por ser biodiverso produce variedad de productos tanto para el consumo interno como externo. Entre los principales productos que exporta se encuentra el banano, camarón, atún, cacao, café entre otros, cuyos principales países destino de las exportaciones son Estados Unidos (54\%), Perú (9,9\%), Chile (8,8\%), Panamá (6,6\%), Colombia (5,5\%), Rusia (4,4\%), Alemania y Holanda $(3,3 \%)$ y otros $(5,5 \%)$. De esta manera, el sector agrario cumple un papel fundamental en la economía del país, el cual genera empleo por sus diversas actividades como la ganadería, pesca, caza, agricultura y silvicultura representando en el 2017 el 26,8\% en empleo siendo superior a otras áreas (Fiallo, 2017).

La producción cafetera en Ecuador tiene una gran capacidad debido a sus diversos ecosistemas, su favorable ubicación geográfica principalmente en provincias con altas temperaturas, aunque sus variados cambios climáticos han generado también pérdidas agrícolas lo que causa que el país tenga una economía vulnerable a consecuencia de las variaciones climatológicas, políticas, medidas o estrategias que se lleven a cabo. En la actualidad muchas familias viven de esta producción, lo que ha incrementado mayor competitividad provocando una disminución en el precio internacionalmente. (Jiménez \& Massa, 2015).

La caficultura en Ecuador tiene mucha importancia tanto en la parte económica porque genera divisas en el país, en la parte cultural se encuentra involucrado muchas culturas étnicas y pueblos, en lo ambiental intervienen los sistemas agroforestales que combinan el suelo y el clima que contribuyen en la preservación de la fauna (Ponce et al., 2018).

Para Pozo (2014), las exportaciones de café en sus dos variedades arábiga y robusta reconocidos por su calidad han sido fundamental para la economía del país, en vista que el $90 \%$ de lo que se produce a nivel nacional se exporta aunque no se logra abastecer el mercado internacional, las exportaciones de café representan el 3,34\% exportaciones no petrolera. Sin embargo, en los últimos años ha venido 
sufriendo una baja de producción lo que se incentiva a generar competitividad para incrementar la producción y cumplir con la demanda tanto interna como externa.

La producción de café se encuentra situado en el puesto 19 en el exterior, desde hace dos décadas la producción del café ha venido decayendo de una forma abrupta tendiendo un déficit a la comercialización y mercado a causa de falta en la innovación, uso de tecnología, semillas productivas, bajos créditos provocan caída en el sector cafetero causando un bajo rendimiento y falta de competitividad a nivel internacional en la producción surgiendo escasez, mano de obra elevada, influyen otros factores como enfermedades de la planta como la Roya que genera un producto de baja calidad, para que su rendimiento llegue a tener mejor efectividad y eficacia se debe realizar planes de contingencia o estrategias gubernamentales que ayuden al caficultor obtener mayor oportunidades y satisfacción en el mercado nacional e internacional (Piña, 2017).

El café tiene un rol importante en las exportaciones tanto en la generación de empleo, divisas como en la parte industrial y el comercio siendo uno de los generadores de producción y economía. Su importancia económica se encarga de divisas para el estado, ingresos a las familias, transportistas, comerciantes, exportadores, microempresarios, entre otros. En su importancia social se encarga de generar empleo a las etnias, productores, comerciantes, agroindustria, bienes y servicios (Suárez, 2018).

La producción de café en es una parte importante por su aporta en la economía del país siendo el sector con mayor incidencia en el empleo por sus diferentes actividades a realizar siendo beneficiados un sinnúmero de familias ya que es un producto exportable que genera ganancias. Esta investigación tiene como objetivo analizar la participación del café en las exportaciones agropecuarias mediante el análisis de los datos recopilados del Sistema de Información y Producción Agropecuaria mediante el cual se propone tener datos verídicos para el estudio y toma de decisión en la producción cafetera.

\section{MATERIALES Y MÉTODOS}

La presente investigación se desarrolló en Ecuador, que se encuentra ubicado en América del Sur, el cual limita al norte con Colombia, sur y este con Perú y al oeste con el Océano Pacifico. La investigación presenta un enfoque descriptivo, de tipo documental, corte transversal y no experimental. El diseño es de tipo exploratorio, porque se centra en analizar la participación del café en las exportaciones agropecuarias del Ecuador durante el periodo 2015-2019, en donde se evaluaron las cifras históricas del Sistema de Información y Producción Agropecuaria (SIPA). En investigaciones similares, Vargas et al. (2021) también utilizó un estudio no experimental, de alcance descriptivo, exploratorio y corte transversal para analizar el mercado del café con enfoque de negocio. 
La información recopilada de la base de datos del SIPA respecto a las exportaciones de café en el periodo evaluado fue procesada en una base de datos en Excel para su respectivo análisis e interpretación descriptiva. Los resultados obtenidos se categorizaron en 4 ejes para su respectiva interpretación en figuras; siendo primero el análisis de las exportaciones de café por producto, el segundo según su destino de exportación, tercero por el Bloque Económico, y el cuarto por los valores mensuales de café exportados (USD).

Según Díaz \& Calsadilla (2015), la investigación descriptiva opera cuando se delinean características obtenidas de las investigaciones exploratorias. De la misma forma, la descripción puede efectuarse mediante métodos cualitativos, y en descripciones superiores usando métodos cuantitativos. Es decir, estos últimos tienen como objetivo medir de forma precisa las dimensiones o características descubiertas en las investigaciones exploratorias. En otras palabras, los estudios exploratorios se centran en descubrir, mientras que los descriptivos en medir con la mayor exactitud posible el fenómeno de estudio.

\section{RESULTADOS Y DISCUSIÓN}

Figura 1. Principales destinos de exportación de café 2015-2019
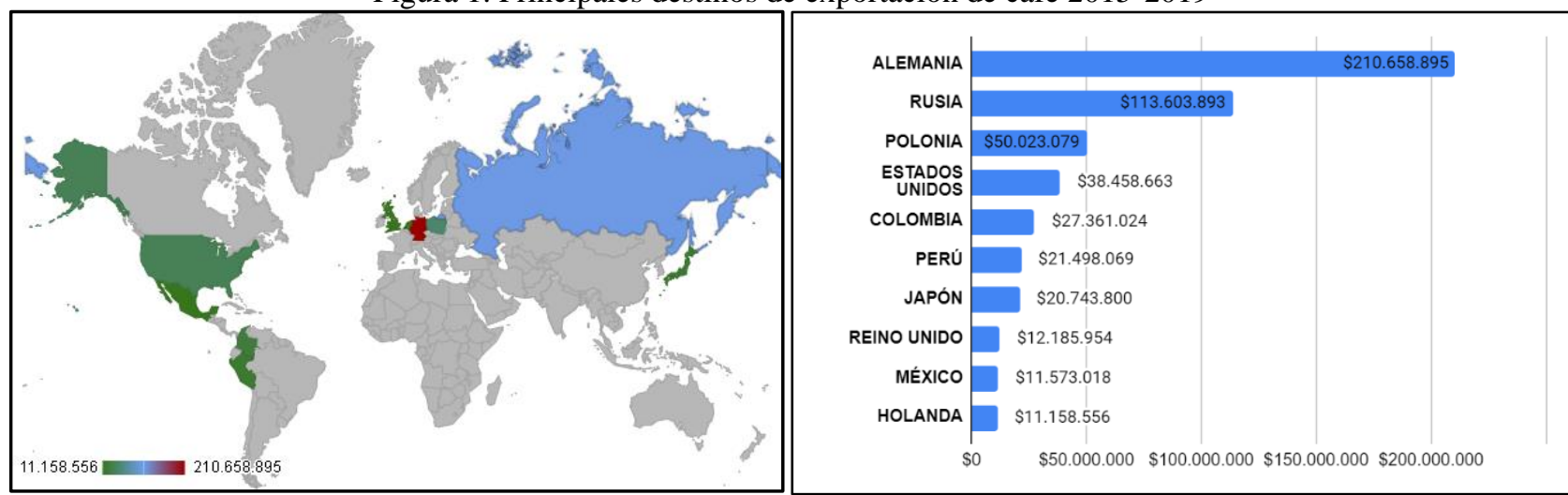

Fuente: SIPA 2015 - 2019

Elaborado por: Los autores

En la Figura 1, se denota los principales destinos de exportación de café ecuatoriano, donde los datos revelan que Alemania ocupó el principal destino durante el periodo 2015-2019 registrando un total de USD 210.658.895 millones de dólares que equivalen al 40.73\% de las exportaciones. Por su parte Rusia ha sido el segundo mayor destino con USD 113.603.893 millones de dólares que representan el 21.96\%. Además, se observa en el mapa que destinos como Estados Unidos, Colombia, Perú, Japón, Reino Unido, México y Países Bajos (Holanda) representaron un monto exportado de USD 192.002.163 millones de dólares que representan el $37.31 \%$ del total exportado. 
Estos datos coinciden con Domínguez et al. (2021) del análisis realizado en su artículo sobre el impacto de las exportaciones e importaciones del Ecuador, donde concluyen que más del $95 \%$ de la oferta exportable del país comprende productos primarios y sus derivados, y los principales destinos de estos productos lideran países como Estados Unidos, Alemania, Países bajos, entre otros.

Tabla 1. Exportaciones de café por Bloque Económico periodo (USD) 2015-2019

\begin{tabular}{|c|c|c|c|c|c|c|c|c|}
\hline$\underset{1}{\text { CCEAG }}$ & $\begin{array}{c}\text { MERCO } \\
\text { SUR }^{2}\end{array}$ & $\underset{3}{\text { ANSA+3 }}$ & $\mathbf{U E E}^{4}$ & TLCAN $^{5}$ & $\mathrm{CAN}^{6}$ & $\mathbf{U E}^{7}$ & AELC $^{8}$ & $\begin{array}{c}\text { CARIC } \\
\text { OM }^{9}\end{array}$ \\
\hline $\begin{array}{c}1.610 .31 \\
5\end{array}$ & 819.353 & $\begin{array}{c}22.543 .0 \\
27\end{array}$ & $\begin{array}{c}113.603 . \\
893\end{array}$ & $\begin{array}{c}50.467 .6 \\
54\end{array}$ & $\begin{array}{c}48.859 .0 \\
93\end{array}$ & $\begin{array}{c}292.456 . \\
418\end{array}$ & 14.419 & 6.718 \\
\hline $0,30 \%$ & $0,15 \%$ & $4,25 \%$ & $21,42 \%$ & $9,52 \%$ & $9,21 \%$ & $55,14 \%$ & $0,003 \%$ & $0,0013 \%$ \\
\hline
\end{tabular}

${ }^{1}$ Consejo de Cooperación para los estados Árabes del Golfo, ${ }^{2}$ Mercado Común del Sur, ${ }^{3}$ Asociación de Naciones del Sureste Asiático más Tres, ${ }^{4}$ Unión Económica Euroasiática, ${ }^{5}$ Tratado de Libre Comercio de América del Norte, ${ }^{6}$ Comunidad Andina, ${ }^{7}$ Unión Europea, ${ }^{8}$ Asociación Europea de Libre Comercio y ${ }^{9}$ Comunidad del Caribe.

Fuente: SIPA 2015 - 2019

Elaborado por: Los autores

De acuerdo a los resultados obtenidos, se puede observar en la Tabla 1 los principales bloques económicos de las exportaciones de café del Ecuador. Se denota que en el periodo 2015-1019 la UE representó el principal Bloque Económico de mayor destino de las exportaciones de café ocupando el 55.14\% de las exportaciones, las cuales registraron un monto de USD 292.456.418 millones de dólares, es decir, 2.57 veces más que la UEE, que fue el segundo Bloque económico con un monto exportado de USD 113.603.893 millones de dólares equivalente al 21,42\% del total exportado.

El TLCAN, CAN representaron el 9,52 y 9,21\% respectivamente de las exportaciones, en donde el monto de ambos bloques sumó un total de USD 99.326.747 millones de dólares. En cambio, la CARICOM, AELC, CCEAG, MERCOSUR y ANSA+3, fueron los Bloques de menor destino de las exportaciones representando el 4.70\% del total exportado con un monto de USD 24.993.832 millones de dólares.

En una investigación similar Mesías (2019) evaluó el comportamiento de las exportaciones ecuatorianas por bloque económico, donde encontró que parte importante de la oferta exportable de los productos ecuatorianos está compuesta por la exportaciones de banano, camarón, flores, atún, cacao, café, entre otros. Y los principales bloques económicos de mayor destina son; la Unión Europea, Estados Unidos, Asociación Latinoamericana de Integración, resto de América y Asia. 
Figura 2. Exportaciones por producto 2015-2019

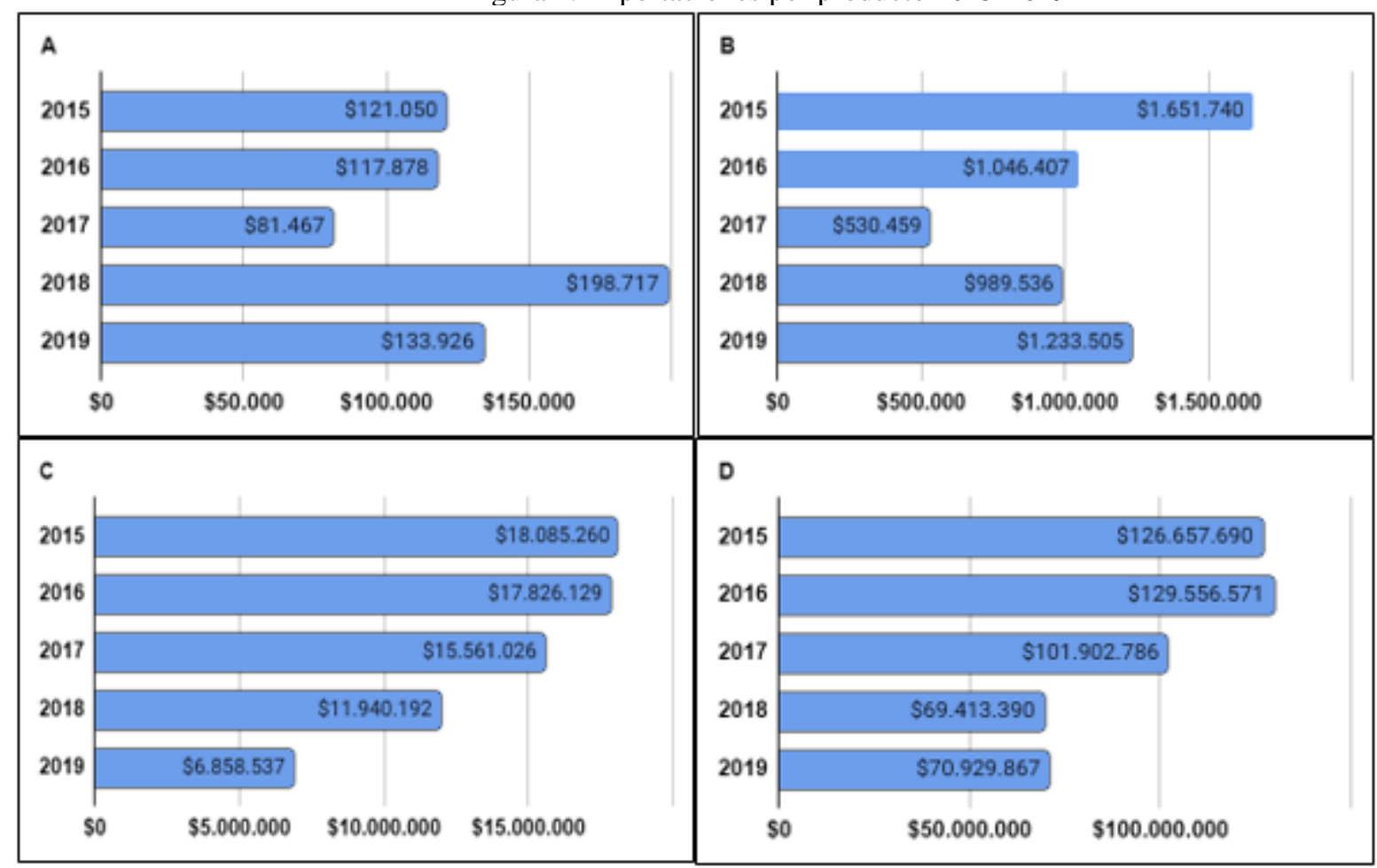

${ }^{A}$ Café tostado descafeinado, ${ }^{\mathrm{B}}$ Café tostado sin descafeinar, ${ }^{\mathrm{C}}$ Café sin tostar sin descafeinar y ${ }^{\mathrm{D}}$ Extractos, esencias y concentrados de café.

Fuente: SIPA 2015 - 2019

Elaborado por: Los autores

Los resultados de las exportaciones por principal producto se denotan en la Figura 2. En el (cuadrante A), que se refiere a café tostado descafeinado se aprecia que la participación de las exportaciones de este producto representa el $0.11 \%$ del total exportado, además que disminuyeron constantemente a una tasa de variación promedio anual del 16,75\% pasando de USD 121.050 dólares en el año 2015, a USD 81.467 dólares en 2017. En cambio, en el año 2018 se registró el mayor valor exportado alcanzado USD 198.717 dólares, sin embargo, para el año siguiente (2019) el monto exportado descendió en un $32.60 \%$ respecto al año anterior cuyo monto exportado fue de USD 133.926 dólares.

Las exportaciones de café tostado sin descafeinar (Cuadrante B) representaron el $0.95 \%$ de las exportaciones por producto en el periodo evaluado, y en los cinco años estas reportaron un total de USD 5.451.647 millones de dólares, siendo el año 2015 (USD 1.651.740) y 2019 (USD 1.233.505) los de mayor valor exportado. De la misma forma, el cuadrante C y D que son del café sin tostar sin descafeinar y extractos, esencias y concentrados de café, son los que mayor participación presentaron en las exportaciones ocupando el $12.22 \%$ y $86.71 \%$ respectivamente.

El producto C (Café sin tostar sin descafeinar) registró un total de USD 70.271.144 millones de dólares cuyo valor exportado presentó una disminución constante del 19,99\% promedio anual desde el 2015 hasta el 2019. Por su parte, el producto D (Extractos, esencias y concentrados de café) reportó por 
concepto de exportaciones un monto de USD 498.60.304 millones de dólares, las cuales en el año 2018 (USD 69.413.390) y 2019 (USD 70.929.867) presentaron una disminución cercana al 30\% respecto a los años anteriores evaluados.

Según el BCE (2020) en su reporte de coyuntura del sector agropecuario coincide con los resultados obtenidos sobre la disminución reflejada de las exportaciones de café por tipo de producto. El reporte destaca que la producción de café en los periodos evaluados (2015-2018) registró una tendencia negativa de $6 \%$ y $8 \%$ en 2018. Entre las principales causas de las cifras negativas reportadas están; las malas condiciones de las plantaciones por la afectación de la roya (8\%), precio del producto (30\%), tecnificación (23\%), factores climáticos (15\%), costos, escasez en mano de obra (8\%) vías de comunicación (8\%) déficit en demanda de producto $(8 \%)$.

Debido a que el precio del café ha sido la principal causa de la disminución de valores de las exportaciones en los últimos años, en el reporte del mercado mundial de café y su impacto en Colombia de Cano et al. (2012) ya sostuvo que, mundialmente los precios a lo que se relaciona con producción agrícola se ven afectadas a causa de subida de precio en combustibles, transportes, fertilizantes aumentando el ingreso per cápita en países desarrollados. De esta manera la disminución en los precios trae consigo afectaciones que afectan el abastecimiento a la demanda a nivel mundial.

Figura 3. Valores de las exportaciones anuales, mensuales y acumuladas de café (USD) 2015-2019

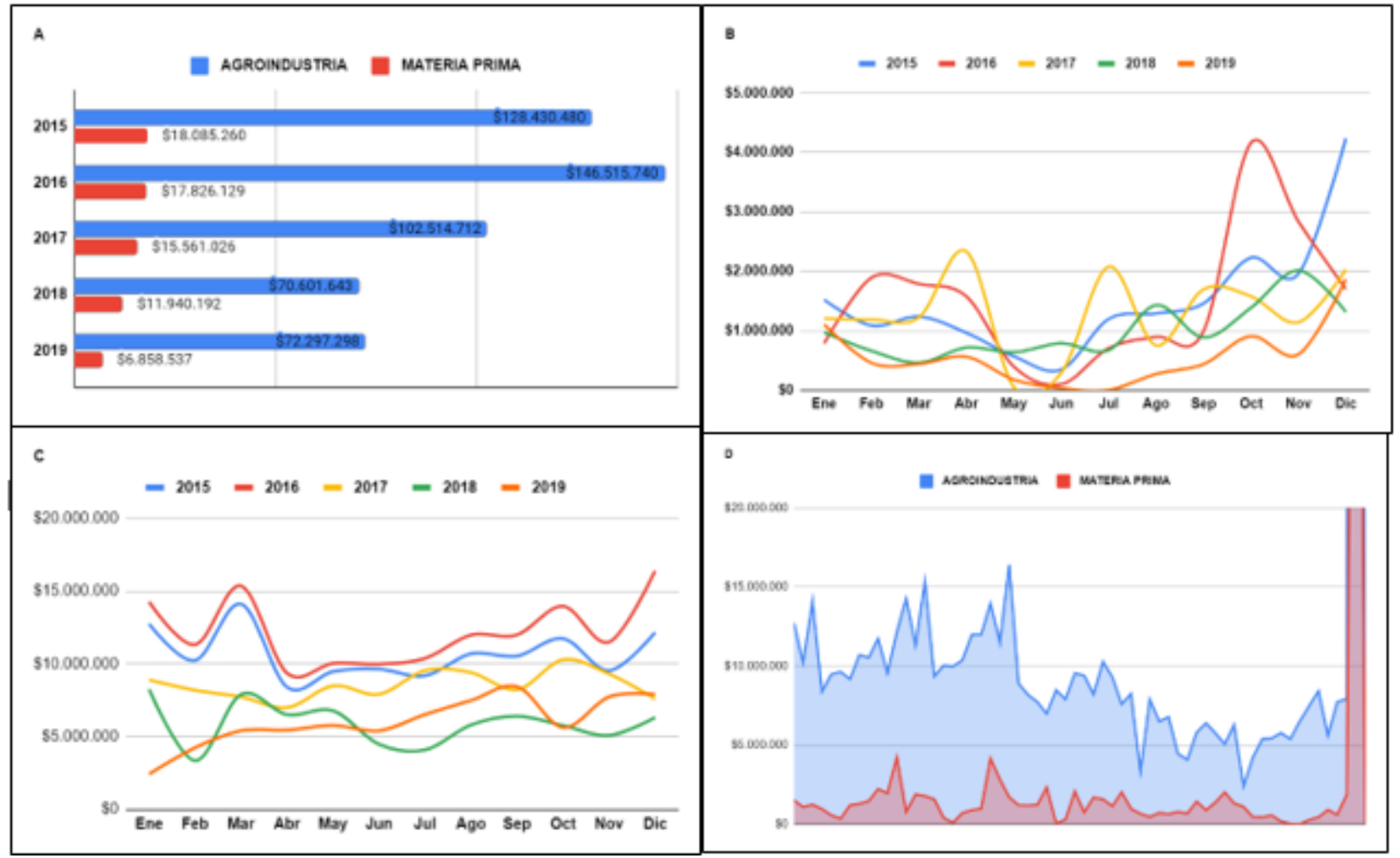

A Valores anuales de café como producto primario y como producto de agroindustria, ${ }^{\mathrm{B}}$ Valores anuales y mensuales de café como producto primario, ${ }^{\mathrm{C}}$ Valores anuales y mensuales de café como producto de agroindustria, ${ }^{\mathrm{D}}$ Valores acumulados de las exportaciones de café durante el periodo 2015-2019. 
Fuente: SIPA 2015 - 2019

Elaborado por: Los autores

En la Figura 3A, se puede visualizar que las exportaciones de café como materia prima tuvieron un decrecimiento promedio del 19,99\% anual. En cambio, como producto de la agroindustria presentaron un crecimiento del 14,08\% en el año 2016 (USD 146.515.740) respecto al año 2015 (USD 128.430480), y a partir del año 2016 hasta el 2019 el valor de las exportaciones como producto industrializado presentaron un decrecimiento promedio anual del 19,58\%. Estos datos coinciden con el Análisis del sector cafetero ecuatoriano de (Sánchez et al., 2019), que analizó el valor de las exportaciones de café como producto primario e industrializado del periodo 2010-2019 obteniendo una tasa de variación promedio similar a la obtenida en la investigación.

Se visualiza en la Figura 3B y 3C los valores mensuales de cada año evaluado del café como materia prima y producto de la agroindustria. Se denota en ambos tipos de producto que las exportaciones han disminuido cada año, siendo el año 2019 para la materia prima de menor valor monetario, mientras que producto de la agroindustria el año 2018 y 2019 son las más bajos. En cambio, los años 2015 y 2016 figuran como los más dominantes registrando los valores exportados más elevados para ambos productos.

Finalmente, en la Figura 3D, se observan los valores acumulados de las exportaciones de café de los últimos 5 años, en donde se denota que la participación de las exportaciones de café en materia representó el 11,90\% mientras que las de la agroindustria el 88,10\% de un monto total entre ambos rubros de USD 590.631.017 millones de dólares. En la Figura también se aprecia la evolución de los valores exportados, donde se observa que la agroindustria presentó su mayor valor exportado en los primeros años (2015-2016). Por su parte, el café exportado en materia prima ha presentado un rendimiento constante y el valor exportado más bajo se evidencia a mediados del último año.

De acuerdo a los resultados obtenidos por Ponce et al. (2018) en su artículo "Situación de la caficultura ecuatoriana: perspectivas" explican que los principales problemas que han provocado que las exportaciones de café cayeran en los últimos años son: baja producción nacional debido a: Prevalencia de cafetales viejos; nivel de productividad bajo; calidad e inocuidad baja; disminución de plantaciones; escaso apoyo como asistencias y capacitaciones técnicas; condiciones climáticas; baja asociatividad; nula innovación tecnológica; carencia de programas para el cultivo, causas que han ocasionado las siguientes consecuencias: Caída de las exportaciones; menor ingreso de divisas; importación; salida de divisas; disminución de ingresos; abandono de los cultivos. 


\section{CONCLUSIONES}

Se concluye que los principales destinos de exportación del café ecuatoriano han sido Alemania y Rusia ocupando más del $60 \%$ de las exportaciones, mientras que destinos como Estados Unidos, Colombia, Perú, Japón, Reino Unido, México y Países Bajos (Holanda) representaron el 37.31\% restante.

Los resultados revelaron que el principal bloque económico de mayor destino de las exportaciones de café en el periodo 2015-1019 fue la UE ocupando el 55.14\% cuyo monto exportado fue 2.57 veces más que las exportaciones hacia la UEE, que fue el segundo bloque de mayor destino representando el 21,42\% del total exportado.

Se obtuvo que los principales productos exportados fueron café tostado sin descafeinar ocupando el $0.95 \%$, café sin tostar sin descafeinar y extractos, esencias y concentrados de café ocupando el $12.22 \%$ y $86.71 \%$ respectivamente, y el café sin tostar sin descafeinar cuyo valor exportado presentó una disminución constante del 19,99\% promedio anual siendo así el producto de más baja representación con $0.12 \%$.

Finalmente se evidenció, que las exportaciones de café como materia prima ocuparon el 11,90\% y las de la agroindustria el $88,10 \%$, además que estas tuvieron un decrecimiento promedio del 19,58\% anual a lo largo del periodo evaluado. Se analizo que las causas de este decrecimiento se dieron por; enfermedades en las plantaciones provocadas por la roya, precio del producto, tecnificación, factores climáticos, costos, escasez en mano de obra, vías de comunicación, y déficit en demanda de producto. 


\section{BIBLIOGRAFÍA}

BCE. (2020). Reporte de conyuntura Sector Agropecuario. Banco Central del Ecuador. https://contenido.bce.fin.ec/documentos/PublicacionesNotas/Catalogo/Encuestas/Coyuntura/Integradas/ etc201904.pdf

Cano, C., Vallejo, C., Caicedo, E., Amador, J., \& Tique, E. (2012). El mercado mundial del café y su impacto en Colombia. https://doi.org/10.32468/be.710

Díaz, V., \& Calsadilla, A. (2015). Artículos científicos, tipos de investigación y productividad científica en las Ciencias de la Salud. Redalyc. https://www.redalyc.org/pdf/562/56243931011.pdf

Domínguez, J., Vega, A., Garzón, V., \& Quezada, J. (2021). IMPACTO OCASIONADO POR LAS EXPORTACIONES E IMPORTACIONES ENTRE ECUADOR Y LA UNIÓN EUROPEA, MEDIANTE EL ACUERDO COMERCIAL MULTIPARTES, PERIODO 2010-2019. Remca. https://remca.umet.edu.ec/index.php/REMCA/article/view/353

Fiallo, J. (2017). Importancia del Sector Agrícola en una Economía Dolarizada (P. Beltrán (ed.)) [Economista, UNIVERSIDAD SAN FRANCISCO DE QUITO USFQ]. https://repositorio.usfq.edu.ec/bitstream/23000/6807/1/134856.pdf

Jiménez, A., \& Massa, P. (2015). Producción de café y variables climáticas: El caso de Espíndola, Ecuador. Redalyc. http://iies.faces.ula.ve/Revista/Articulos/Revista_40/Pdf/Rev40JimenezT.pdf

Mesías, R. (2019). El acuerdo comercial de Ecuador con la Unión Europea y el comportamiento de las exportaciones ecuatorianas (J. Rivera (ed.)) [Maestría en Administración de Empresas, Universidad Andina Simón Bolívar]. https://repositorio.uasb.edu.ec/bitstream/10644/6680/1/T2887-MAE-MesiasEl\%20acuerdo.pdf

Piña, K. (2017). Análisis económico del sector cafetero de la región costa del Ecuador, período 20102014 [Universidad de Guayaquil. Facultad de Ciencias Económicas]. http://repositorio.ug.edu.ec/handle/redug/36741

Ponce, L., Orellana, K., Acuña, I., Alfonso, J., \& Fuentes, T. (2018). Situación de la caficultura ecuatoriana: perspectivas. Revista Estudios Del Desarrollo Social: Cuba Y América Latina , 6(1), 307325.

Pozo, M. (2014). Análisis de los factores que inciden en la producción de café en el Ecuador 2000-2011 [Pontificia Universidad Católica del Ecuador]. http://repositorio.puce.edu.ec/handle/22000/6848

Sánchez, M., Vayas, T., Mayorga, F., \& Freire, C. (2019). Análisis del sector cafetero ecuatoriano. Observatorio Económico Y Social deTungurahua. https://blogs.cedia.org.ec/obest/wpcontent/uploads/sites/7/2020/10/Analisis-del-sector-cafetero-ecuatoriano-final-tres.pdf

Suárez, L. (2018). Análisis de las exportaciones de café y su participación en las exportaciones no petroleras del Ecuador durante el período 2012-2016 [Universidad de Guayaquil. Facultad de Ciencias Económicas]. http://repositorio.ug.edu.ec/handle/redug/29180

Vargas, M., Quezada, J., García, R., \& Carvajal, H. (2021). Análisis de mercado para el procesamiento y comercialización de café tostado y molido, cantón Marcabelí. Revista Metropolitana de Ciencias 
Aplicadas, 4(2), 99-106. 